\title{
BMJ Open Quality Improving the quality of trauma meetings by implementation of a modern trauma management platform
}

\author{
Benjamin Michael Sephton (D) , ${ }^{1}$ Olivia Katherine Vernon, ${ }^{1}$ Kathryn Kimber, ${ }^{1}$ \\ Michael Shenouda, ${ }^{2}$ Piyush Mahapatra ${ }^{3}$
}

To cite: Sephton BM, Vernon OK, Kimber K, et al. Improving the quality of trauma meetings by implementation of a modern trauma management platform. BMJ Open Quality 2020;9:e000998. doi:10.1136/ bmjoq-2020-000998

Received 15 April 2020 Revised 21 June 2020 Accepted 29 July 2020
Check for updates

(C) Author(s) (or their employer(s)) 2020. Re-use permitted under CC BY-NC. No commercial re-use. See rights and permissions. Published by BMJ.

${ }^{1}$ Orthopaedics, University Hospital of South Manchester NHS Foundation Trust, Manchester, UK

${ }^{2}$ Orthopaedics, Hillingdon Hospital NHS Trust, Uxbridge, UK ${ }^{3}$ Orthopaedics, Imperial College Healthcare NHS Trust, London, UK

Correspondence to Dr Benjamin Michael Sephton; b.sephton@nhs.net

\section{ABSTRACT}

Trauma meetings constitute the majority of clinical decision-making for new referrals and also act as a crucial tool to coordinate the trauma theatre list. Incorporation of electronic technology has been shown to improve the quality of patient handover. eTrauma is a recently developed cloud-based patient management platform which we planned to implement at our hospital. The aim of our project was to evaluate the quality of our current trauma meeting and subsequently the effect of implementing a modern trauma management platform. In order to identify the key concerns of staff members with the trauma meeting handover, a questionnaire was given to 20 members of the team. A review of effective handover guidelines published from the Royal College of Surgeons and the Royal College of Physicians was undertaken. We combined information from these guidelines to identify two key sets of data points for an effective trauma referral: patient demographics and referral details. Questionnaires were distributed at three separate time periods providing assessment of implementation of changes. The questionnaire results indicated progressive improvement in the morning trauma meeting with increased clarity for the anaesthetic team, better communication of operation to be performed, better documentation and clarity of management plans. A total of 150 referrals using the existing system (TIGER) were compared with 100 eTrauma referrals at two separate time intervals. Our analysis showed significant improvements in 12 (57\%) of the 21 key data points indicating improved data entry for referrals using the new platform. Three PDSA (Plan, Do, Study, Act) cycles were completed with relevant adjustments within this process. Introduction of a new cloud-based trauma management platform has had a positive impact overall within our trust. Modern electronic trauma systems have the ability to improve our trauma management, this must go hand in hand, however, with a structured and effectively communicated trauma meeting.

\section{PROBLEM}

Approximately 1.8 million fractures occur per year in England and 30\% of all emergency department attendances are related to musculoskeletal injuries. ${ }^{12}$ Around $20 \%$ of these injuries will be referred onwards to the on-call orthopaedic team for urgent review. ${ }^{1}$ Wythenshawe hospital is a major acute teaching hospital in South Manchester.
The Trauma and Orthopaedic Department consists of approximately 12 consultants; with around 50-60 orthopaedic inpatients on an average day. The previous trauma referral system involved a clinician-built computer application, known as TIGER. The system allows to input the date of referral, location of the patient, responsible consultant and a free-text box for entering the referral details. The referred patients are then split into Trauma List patients (awaiting an operation) and new referrals (ones unlikely to be operated on but need to be discussed).

On discussion with the orthopaedic team (primary users), the consensus was that while this system had functioned for a number of years, it did have some significant drawbacks. First, the free-text entry meant that particular aspects of the referral history may be missed; for example, social history, mobility, smoking and allergy status. This meant that the quality of new referrals was not ensured. There was also the ability to overwrite the previous free-text entry, which is evidently not acceptable from a governance or medicolegal perspective.

A further, considerable problem was the organisation of the trauma list. With patients placed in chronological order on the 'Trauma List' section it was not possible to establish the order of the trauma list and which patients should be prioritised to be seen or operated on first. This may have led to delays in starting times and between cases, potentially incurring substantial costs to the trust through loss of valuable theatre time. Finally, documentation of the plan and outcome from the trauma meeting was unclear with a post-hoc entry added to the end of the free-text data entry for each patient, by the trauma nurses.

With the fast pace of trauma meetings, it is essential to have an accurate, highquality referral with all relevant information included. It is then imperative that theatre staff including anaesthetists and scrub nurses 
Table 1 Patient demographics and referral details data points used for comparative analysis

\begin{tabular}{ll}
\hline Patient demographics & Referral details \\
\hline 1. Patient name & 1. Diagnosis \\
\hline 2. Date of birth & 2.Laterality \\
\hline 3. NHS/hospital number & 3.Mechanism of injury \\
4. Date of referral & 4.Examination findings \\
\hline 5. Ward location & 5.Past medical history \\
6. Bed allocation & 6.Allergies \\
7. Responsible consultant & 7.Relevant drug history \\
& 8.Blood results \\
& 9.Investigation results \\
& 10.Baseline mobility \\
& 11.Walking aid \\
\hline & 12. Handedness (if relevant) \\
\hline & 13.Occupation \\
\hline
\end{tabular}

NHS, National Health Service.

have a clear idea of the order of the trauma list and which patients to prioritise. The outcome of the trauma meeting should be clearly documented allowing patients to be managed in a safe manner. Failure to follow this process has led to potential delays in service provision, confusion regarding plans and may have negatively impacted on patient care.

The primary SMART aim of our project was to improve the quality of electronic documentation of trauma and orthopaedic referrals at our institution. To provide a quantitative measure of referral quality we developed 21 key data points (table 1) that are needed for a good orthopaedic referral including all relevant information to allow further assessment. At baseline, 12 of 21 key data points were documented in $<80 \%$ of referrals indicating major room for improvement. We aimed to a show significant improvement in $50 \%$ of key data points within a 6-month period.

Our secondary SMART aim was to improve the structure and patient handover at our morning trauma meeting. A qualitative questionnaire was distributed at separate time points looking at four key areas of the trauma meeting: structure of the trauma meeting, clarity of the daily trauma list, clarity and documentation of operations to be performed and clarity and documentation of management plans. We aimed to show an improved response in $75 \%$ of our qualitative feedback questions over 6 months indicating better coordination of the patient handover at the trauma meeting.

\section{BACKGROUND}

Trauma meetings constitute the majority of clinical decision-making for new referrals and also act as a crucial tool to coordinate the trauma theatre list. The General
Medical Council, an independent regulator for doctors within the UK, states 'A well-managed, thorough and organised handover is crucial for ensuring the quality and safety of patient care'. ${ }^{3}$ It is an area, which if done poorly, can potentially compromise patient safety and may result in patient harm. ${ }^{45}$

The National Health Service (NHS) institute for innovation and improvement has calculated that trusts can save $£ 7$ million a year in efficiency savings from running a 'productive theatre'. ${ }^{6}$ Late start times are a common reason for operative time lost on the trauma or emergency list across all surgical specialities. ${ }^{7}$ A delay of 1 hour at the start of the trauma list is estimated to cost a trust around $£ 1400 .{ }^{8}$ While reasons for delays are multifactorial, clarity of list order at the trauma meeting is a significant contributing factor.

Incorporation of electronic technology has repeatedly been shown to improve the quality of patient handover. ${ }^{9-13}$ The British Medical Association states that 'systems need to be put in place to enable and facilitate handover. These systems, although based on a generic model, must be adapted to local needs'. ${ }^{14}$ eTrauma (Open Medical Ltd, UK) is a recently developed cloud-based patient management platform which is used to process all new orthopaedic referrals as well as coordination of operative trauma lists. Modern trauma management platforms, such as eTrauma, aim to provide clearer handover instructions, improve patient flow and allow interhospital communication.

\section{BASELINE MEASUREMENT}

The orthopaedic management team had decided to search for a new trauma platform in light of the aforementioned issues with the existing system. The key drivers for implementation of change are outlined in figure 1 . In order to confirm the informal issues with the trauma meeting handover, a questionnaire was given to 20 members of the team including orthopaedic trauma nurses, foundation year doctors, core surgical trainees, registrars and consultants. The questionnaire utilised Likert scales for subjective qualitative assessment and provided a baseline measurement for comparison (figure 2). The questionnaire focused on the following four key aspects:

1. Structure of trauma meeting.

2. Clarity of daily trauma list.

3. Clarity and documentation of operations to be performed.

4. Clarity and documentation of management plans A review of effective handover guidelines published from the Royal College of Surgeons ${ }^{15}$ and the Royal College of Physicians ${ }^{16}$ was undertaken. We combined information from these guidelines and our own trauma meeting experience allowing development of two key sets of data points for an effective trauma referral which were patient demographics and referral details (table 1). An analysis of 150 consecutive trauma referrals was carried out dating from 12 to 22 September 2019 and referrals were compared 


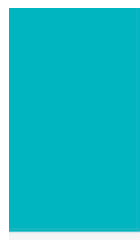

\section{Primary Drivers Secondary Drivers}

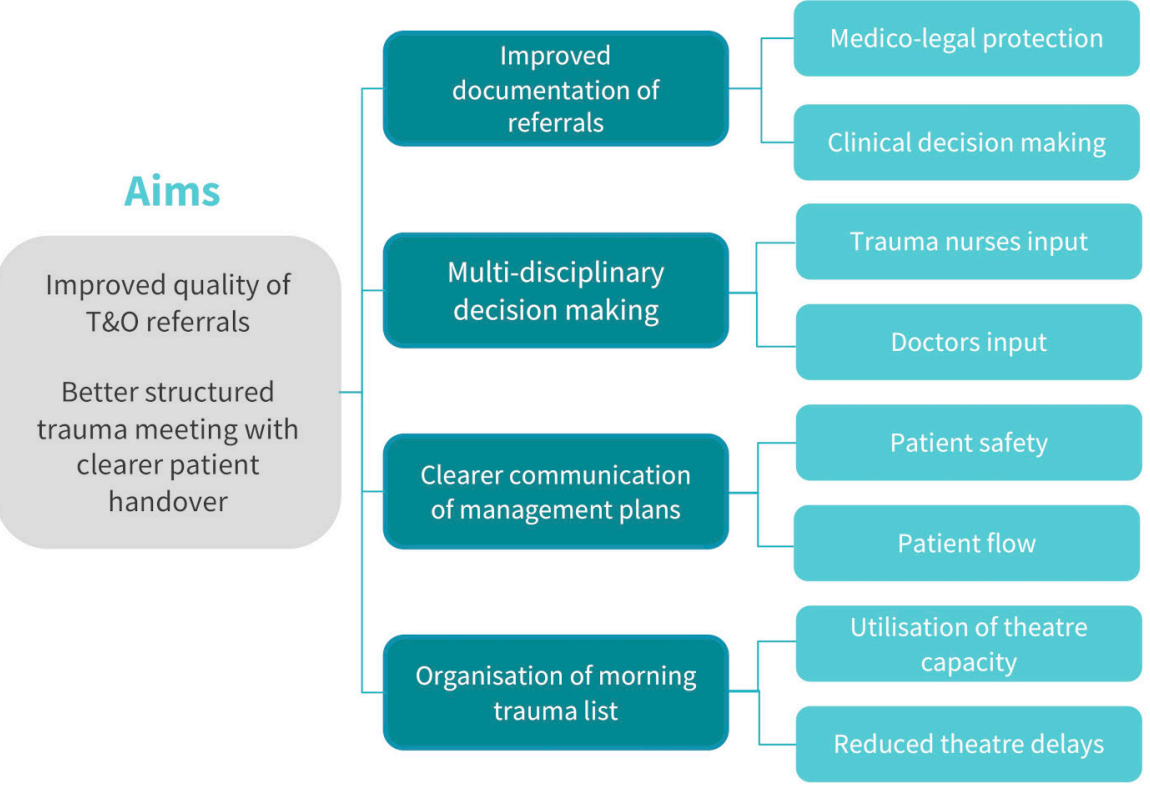

Change Ideas

$\begin{gathered}\text { Encourage completion of } \\ \text { pre-set data fields }\end{gathered}$
Utilisation of electronic
system features
Team education with new
system
All team members
contribute to meeting
Documentation of plans
after meetings
Accurate coding of
planned operation
Live time tracking of
changes to trauma list
Ensure first patient on list
is clearly communicated

Figure 1 Key drivers for implementation of change.

with our key data points. This provided us with a quantitative assessment of electronic trauma referral data entry for further analysis.

\section{DESIGN}

The planned intervention was to introduce a modern trauma management system to help better coordinate our trauma referrals and plan our theatre list. eTrauma is a cloud-based platform that provides real-time data entry to facilitate optimisation of trauma services with overall operational costs of $£ 15000$ per annum. With regards to inputting referrals, there are prompted data entry fields such as demographics, responsible consultant, mechanism of injury, social background and mobility status in which referrers can input data (figure 3). At the same time there are coded entries for past medical history, allergies and drug history as well as diagnosis which gives ability to audit practice through accurate coding. The overall aim of these preprompted fields is to improve the quality of referrals and increase data capture.

Another heralded advantage of the new trauma system was the ability to coordinate the trauma list in real time. The interactive system allows tracking of trauma list order and entry of procedure to be performed, expected duration of the operation and what equipment is needed. This theoretically would allow us to edit the trauma list in realtime, while also allowing planning for days later in the week-thus improving operative planning and trauma patient flow.
Following contract agreement with the eTrauma team, an implementation date of 30 September 2019 was set. The team migrated existing data onto the new system the day before and customised the pathways to fit our specific hospital (ward names, current referral systems, etc).

\section{STRATEGY}

\section{PDSA cycle 1}

Aim

To establish team members opinions regarding the current trauma meeting structure by distribution of an eight-part questionnaire (figure 2). Alongside this we wanted to establish a baseline measurement of the existing system, measuring documentation of referrals against our key data points, as outlined in table 1 . Following collection of baseline measurements, we aimed to successfully introduce the new eTrauma system.

\section{Strategy for change and implementation}

Questionnaires were distributed to members of the orthopaedic department ranging from trauma nurses, foundation year doctors, core and specialty trainees and consultants. We analysed 150 consecutive referrals from the TIGER system against our key data points and documented results for further comparison. Members of the trauma and orthopaedic team were given a presentation and demonstration of the trauma pathway on the introduction date. A member of the eTrauma team also provided on-site support throughout the first week to aid transition; while the on-call team had access to 24-hour 


\section{Improving Trauma Handover - Questionnaire}
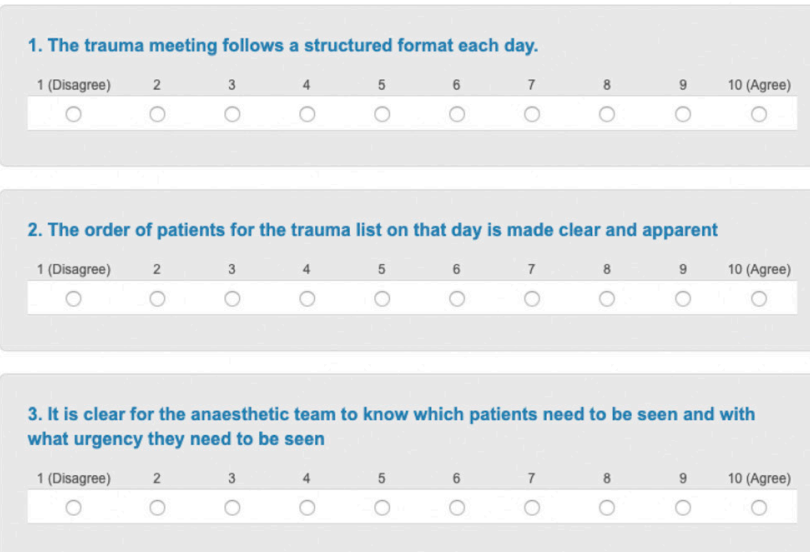

4. The operation decided for each patient is clearly communicated

$\begin{array}{llllllllll}1 \text { (Disagree) } & 2 & 3 & 4 & 5 & 6 & 7 & 8 & 9 & 10 \text { (Agree) }\end{array}$

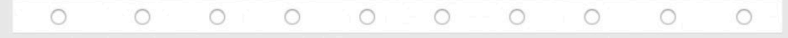

5. The operation decided for each patient is clearly documented

$\begin{array}{cccccccccc}1 \text { (Disagree) } & 2 & 3 & 4 & 5 & 6 & 7 & 8 & 9 & 10 \text { (Agree) } \\ 0 & 0 & 0 & 0 & 0 & 0 & 0 & 0 & 0 & 0\end{array}$

ran

6. The plan for new referrals is clear

$\begin{array}{llllllllll}1 \text { (Disagree) } & 2 & 3 & 4 & 5 & 6 & 7 & 8 & 9 & 10 \text { (Agree) }\end{array}$

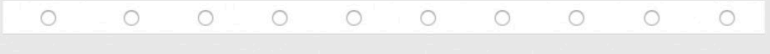

7. The plan for new referrals is documented

\begin{tabular}{llllllllll}
1 (Disagree) & 2 & 3 & 4 & 5 & 6 & 7 & 8 & 9 & 10 (Agree) \\
\hline & 0 & 0 & 0 & 0 & 0 & &
\end{tabular}

8. Any chances to management plan / list order are clearly communicated

$\begin{array}{llllllllll}1 \text { (Disagree) } & 2 & 3 & 4 & 5 & 6 & 7 & 8 & 9 & 10 \text { (Agree) }\end{array}$

Figure 2 Questionnaire regarding structure, clarity and communication of trauma meeting.

online troubleshooting. Finally, we distributed a comprehensive guide and posters for using the eTrauma system which was provided to all junior doctors.

\section{Data and key learning}

Initial responses from the questionnaire showed areas for improvements notably in the clarity plus documentation of management plans and operations to be performed. As predicted both our questionnaire and audit of new referrals suggested improvements could be made with both the trauma referral data input and for the conduction of the current trauma meeting. Overall the referral quality for our demographic data points was well documented. However, the key referral details were considerably lacking, particularly in areas regarding drug history and allergy status as well as documentation of all aspects of social history. Authors felt these findings indicated that there was room for improvement.

The transition to the new system went successfully, of particular value was the on-site support, for the first 7 days. There was regular demonstration of the new systems features at the morning meeting from the open medical team. The trauma nurses appeared to adapt to the new system quickly with regular usage of eTrauma and scheduling of patients onto the trauma list. There seemed to be more confusion once the eTrauma team members were no longer on site. Junior doctors often only had exposure to the system when they were on call and due to the large number of senior house officers on the rota, it meant that doctors gained incremental experience of the system more slowly.

\section{PDSA cycle 2}

Aim

To analyse data points for comparison with baseline measurements we planned to redistribute the eight-part questionnaire to the same 20 members of the trauma team. We also aimed to compare compliance with our initial 21 key data points. Following implementation, we allowed an initial adjustment period of 1 month prior to further analysis of the new system.

\section{Change hypothesis}

It was hypothesised that implementation of the new system would improve the documentation and therefore quality of new referrals. It was hoped that the predetermined data entry fields would encourage junior doctors to provide a complete history and improve the compliance against our key data points.

\section{Data}

One month after the introduction of the new system the same eight-part questionnaires were distributed to 20 members of the orthopaedic team. We then audited 100 consecutive trauma referrals using the eTrauma platform against the same 21 key data points. Our analysis showed significant improvements in $12(57.1 \%)$ of the 21 key data points indicating improved data entry for referrals using the new trauma management platform (eTrauma). The analysis from the questionnaires, however, showed limited improvement in the structure of the trauma meeting and the documentation and clarity of patient management plans.

\section{Strategy for change \& implementation}

Following collection of the results, we presented our initial findings at our regional audit and clinical evaluation meeting at Trafford General Hospital. This occurred around 2 months postintroduction of the new system. We noted feedback from consultants and other members of the trauma team and drew relevant conclusions to make further improvements. The majority of the feedback concerned the structure of the daily trauma meeting and organisation of printing of the handover list. 


\section{eTீrauma}

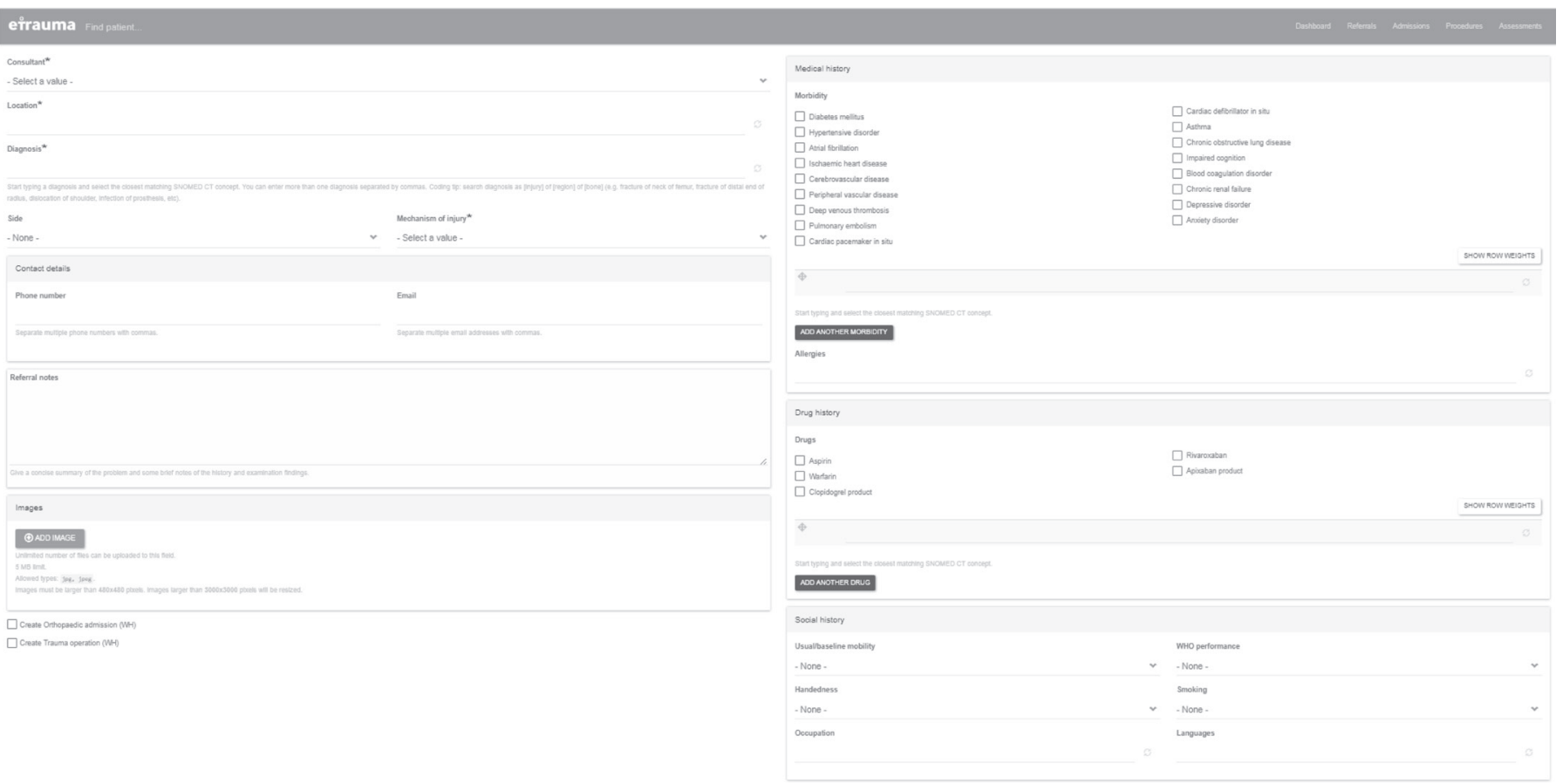

Figure 3 eTrauma data entry fields.

\section{Key learning}

While the results are potentially explained by unfamiliarity with the new system and resistance to change, it was clear from the initial results that some key areas needed to be targeted for further improvement. We identified that we needed to improve the structure of our trauma meeting, the clarity of the trauma list and documentation of management plans. We constructed two key strategies to use the new systems' features and improve our trauma meetings with the feedback received from the regional meeting.

\section{PDSA cycle 3}

Aim

Our aim was to make several key changes over the next 2 months prior to reauditing to see if these changes made any impact on our key data points and questionnaire feedback. We aimed to gradually incorporate changes by ensuring regular discussion and reminders to colleagues regarding the changes outlined below.

\section{Change hypothesis}

The following hypotheses were made. First, uniform list printing would lead to better and more familiar structure to the morning meeting. Second, encouraging documentation of management plans by highlighting of this function to team members, would improve questionnaire responses regarding this topic. Third, we hypothesised that familiarity with the system and presentation of issues at the previous audit meeting would lead to increased compliance with our key data points representing improvements to the quality of incoming referrals.

\section{Strategy for change and implementation}

Our first strategy was to ensure the structure of the trauma meeting was uniform. The trauma meeting structure is often dictated by trauma list printing, there are four individual list sections to be printed each day which resulted in confusion as these lists were not collated in the same order each day. Therefore, following a discussion with trauma nurses and team members (responsible for list printing), we agreed on a uniform structure for the morning meeting with presentation of the trauma list followed by new admissions then by new referrals (that were not admitted) and finally postoperative reviews.

Our second strategy was to encourage documentation of the trauma meeting outcomes for all referred patients. Again, this was discussed with trauma nurses and junior doctors with agreement that the individual operating a second computer would update the outcome of the trauma meeting as the referral was processed. This was relatively easy to incorporate as we were able to add a comment on the eTrauma system for each referral which was then saved for future reference.

\section{Data}

Finally, we redistributed questionnaires and analysed a further 100 trauma referrals against the same key data points, 4 months postintroduction of the new trauma system. The aim of this was to evaluate the impact of the trauma system when members of the orthopaedic team were familiar with inputting referrals and able to fully use the features of the new platform. Our analysis again showed significant improvements in $12(57 \%)$ of 
the 21 key data points indicating improved data entry for referrals. The questionnaire results indicated progressive improvement in the morning trauma meeting with increased clarity for the anaesthetic team, better communication of operation to be performed plus better documentation and clarity of management plans.

\section{Key learning}

Clarifying the list order made a huge difference. This allowed team members a more familiar trauma meeting configuration similar to that used with the previous system. This was coupled with increased clarity of the trauma list with the list for that day displayed both on screen and the printed handouts. The documentation of the management plan worked incredibly well, trauma nurses updated plans post the trauma meeting which was clear and apparent on the system as well as being shown on the printed list the following day. One area which caused an issue was in documentation of the operation to be performed. Although junior doctors often completed this data entry field, the numerous different codes for each possible operation meant this was often incorrect and needed to be changed within the trauma meeting. This was noted and junior doctors were encouraged to code the designated operation as accurately as possible.

\section{Patient and public involvement}

This project was done without patient involvement. Patients were not invited to comment on the study design and were not consulted to develop patient relevant outcomes or interpret the results. Patients were not invited to contribute to the writing or editing of this document for readability or accuracy.

\section{RESULTS}

Our investigation into the impact of the new trauma system was divided into two main strands for assessment: quality of data entry for new referrals and qualitative assessment of the structure and clarity of the trauma meeting.

\section{Quality of data entry for new referrals}

In assessing the quality of data entry for new referrals, we developed 21 key data points for comparison (table 1). These were divided into two subsets of patient demographics and referrals details. We collected data at three stages: PDSA cycle 1 (0 months), PDSA cycle 2 (1 month) and PDSA cycle 3 (4 months). We analysed these groups for significance using Fishers exact test and $\chi^{2}$ test; a $p$ value of $<0.05$ was considered significant. All statistical analyses were performed using XL Stat (Addinsoft, New York, USA).

Patient demographic details were well recorded across both TIGER and eTrauma systems with $100 \%$ of referrals including the following: patient name, date of birth, hospital/NHS number, date of referral, ward location and responsible consultant. Bed allocation (for admitted patients) was not recorded in any patients $(0 \%)$ on the
TIGER system, this improved significantly to $49.2 \%$ and $49 \%$ with eTrauma at 1 and 4 months time points ( $>00.001)$. Patient demographics details are shown in table 2 and figure 4.

Key referral details included 14 data points that constitute a good trauma referral (table 1). Both diagnosis and laterality of injury were recorded in $>94 \%$ of referrals on both trauma systems and there was no significant difference between the two system for these data points. Blood results were poorly documented on the TIGER system at $37.2 \%$ of referrals which showed no improvement at both $1(39.5 \%)$ and 4 months $(38.1 \%)$ postintroduction of the new eTrauma system. The remaining data values all showed significant improvements with the eTrauma system: mechanism of injury $(p>0.001)$, examination findings $(p=0.035)$, past medical history $(p=0.003)$, documentation of allergies $(\mathrm{p}>0.001)$, relevant drug history $(p>0.001)$, investigation results $(p>0.001)$, baseline mobility $(p>0.001)$, walking aid $(p>0.001)$, handedness for upper limb fractures $(p=0.019)$, occupation $(p>0.001)$ and smoking status $(\mathrm{p}=0.015)$. Referral details are shown in table 2 and figure 5 .

\section{Qualitative assessment of the structure and clarity of the trauma meeting}

To assess the impact that introduction of the new system had on the structure and clarity of the trauma meeting. we distributed a questionnaire, as outlined in figure 2. The questionnaire consisted of four key themes including; the structure of the trauma meeting, clarity of the daily trauma list, clarity and documentation of operations to be performed and clarity and documentation of management plans. Utilisation of a Likert scale allowed qualitative data for comparison, ${ }^{17}$ with 1 representing strongly disagree to 10 representing strongly agree. The average mean responses for each question are presented in figure 6 and table 3 .

With regards to the structure of the trauma meeting, responses were initially very positive with the TIGER system suggesting a good pre-existing structure to the trauma meeting. At 1-month postintroduction, responses suggested that the trauma meeting had become subjectively less structured. Authors, as previously stated, attributed this to unfamiliarity of the new system and a lack of established order of list printing. At 4 months, responses were similar to those seen at preintervention level; suggesting the team were more familiar with the system and trauma meetings were again following a set structure.

In terms of the daily trauma list, the clarity of the order of patients on the list remained similar using both systems. However, responses indicated increased clarity for the anaesthetic team with the new system, in knowing which patients needed to be seen first and with what urgency they needed to be seen. Authors feel this was due to the ability to allocate patients a specific position on the trauma list, thereby establishing priority, which was not possible with the existing system. 
Table 2 Differences in patient demographics and key referral details recorded between the two trauma systems

\begin{tabular}{|c|c|c|c|c|}
\hline & $\begin{array}{l}\text { TIGER } \\
\text { (PDSA cycle 1) \% }\end{array}$ & $\begin{array}{l}\text { eTrauma } \\
\text { (PDSA cycle 2) \% }\end{array}$ & $\begin{array}{l}\text { eTrauma } \\
\text { (PDSA cycle 3) \% }\end{array}$ & $P$ value \\
\hline \multicolumn{5}{|l|}{ Patient demographics n (\%) } \\
\hline Date of birth & 100 & 100 & 100 & $1.000^{*}$ \\
\hline Hospital/NHS number & 100 & 100 & 100 & $1.000^{*}$ \\
\hline Bed allocation & 0 & 49.2 & 49 & $>0.001^{*}$ \\
\hline Responsible consultant & 100 & 100 & 100 & $1.000^{*}$ \\
\hline \multicolumn{5}{|l|}{ Referral details n (\%) } \\
\hline Diagnosis & 97.3 & 100 & 100 & $0.098^{*}$ \\
\hline Past medical history & 76 & 88 & 91 & $0.003 \dagger$ \\
\hline Allergies & 6.7 & 23 & 33 & $>0.001 \dagger$ \\
\hline Relevant drug history & 5.3 & 50 & 51 & $>0.001 \dagger$ \\
\hline Blood results & 37.2 & 39.5 & 38.1 & $0.716 \dagger$ \\
\hline Investigation results & 74.6 & 93.9 & 95 & $>0.001 \dagger$ \\
\hline Baseline mobility & 38.8 & 55.7 & 67 & $>0.001 \dagger$ \\
\hline Walking aid & 35.7 & 55.3 & 62.8 & $>0.001 \dagger$ \\
\hline Handedness (UL fractures) & 21.2 & 41.6 & 52.2 & $0.019 \dagger$ \\
\hline
\end{tabular}

Entries in bold indicate statistically significant values.

${ }^{*}$ Fishers exact test.

$+x^{2}$ test.

NHS, National Health Service; UL, upper limb.

Results indicated that the communication of operation to be performed was clearer with the new system. Alongside this the documentation of the operation to be performed was vastly improved at 4 months postintervention compared with the pre-existing Tiger system. This is likely due to the fact that the user must input the planned

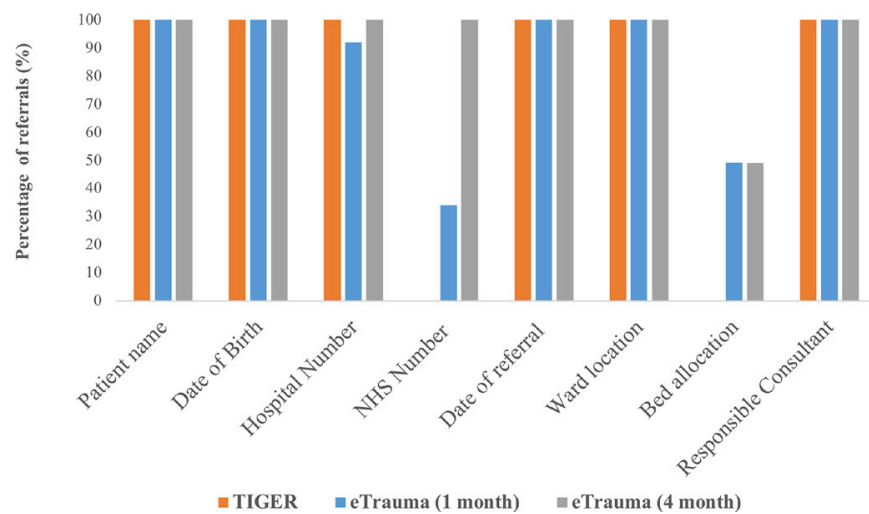

Figure 4 Percentage of referrals documenting patient demographics data points. operation in order to place patients onto the trauma list. Operations could then be edited if the plan was changed. This adjustment differed from the preintervention practice which relied on verbal communication of the planned operation in the morning meeting.

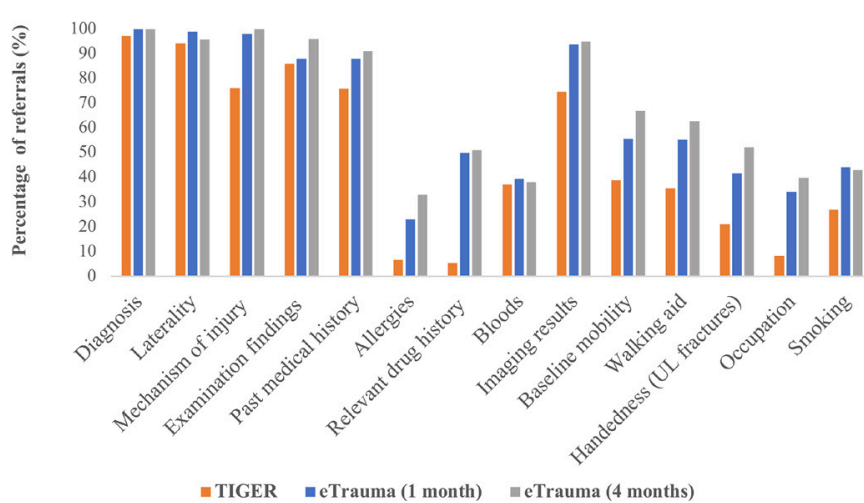

Figure 5 Percentage of referrals documenting key referral details. UL, upper limb. 


\section{meTrauma (4 month) घeTrauma (1 month) —TIGER}

The trauma meeting follows a structured format each day

The order of patients for the trauma list on that day is made clear and apparent

It is clear for the anaesthetic team to know which patients need to be seen and with what urgency they need to be seen

The operation decided for each patient is clearly communicated

The operation decided for each patient is clearly documented

The plan for new referrals is clear

The plan for new referrals is documented

Any changes to management plan / list order are clearly communicated

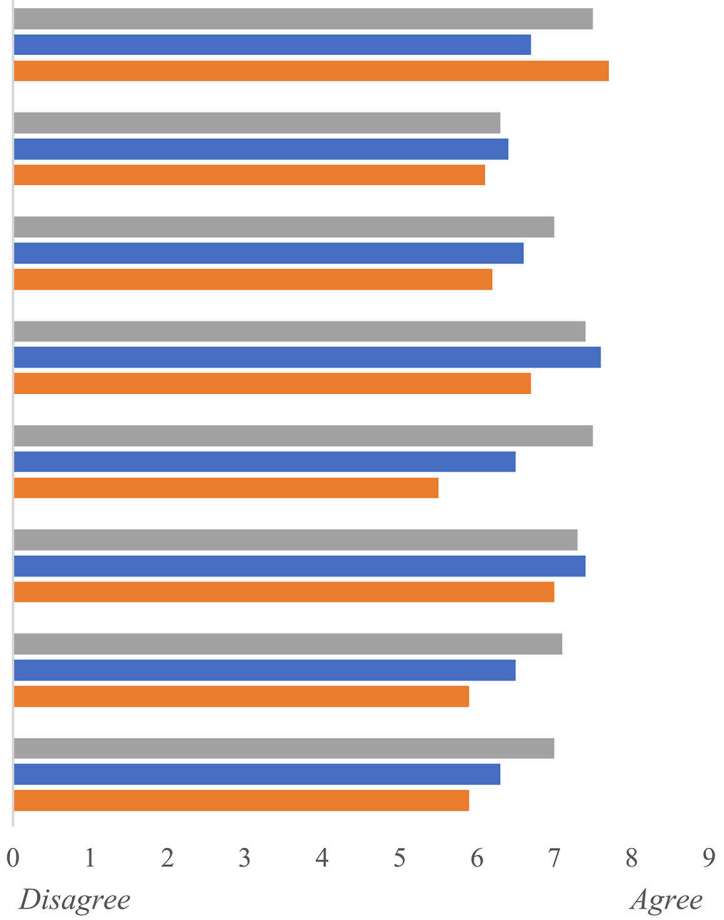

Figure 6 Qualitative analysis of questionnaire results (via average Likert score).

The clarity of the plan for new referrals was similar for both systems; this factor is likely influenced by the quality of the communication skills of the team members rather than the trauma system itself, as discussed below. The documentation of these plans, however, was improved with the eTrauma system in comparison to preintervention responses. Authors feel that one of the key improvements with the new system was the ability to enter comments on each new referral before, during or after the trauma meeting. Thus allowing an updated management plan throughout the patients in-hospital stay. Finally, any changes to management plan and list order appeared to be improved with the new system.

Table 3 Differences in questionnaire results between trauma systems via Likert scale averages

\begin{tabular}{|c|c|c|c|}
\hline & $\begin{array}{l}\text { TIGER } \mathrm{n}=25 \\
\text { (mean, SD) }\end{array}$ & $\begin{array}{l}\text { eTrauma } \\
\text { (1 month) n=25 } \\
\text { (mean, SD) }\end{array}$ & $\begin{array}{l}\text { eTrauma } \\
\text { ( } 4 \text { month) } n=25 \\
\text { (mean, SD) }\end{array}$ \\
\hline \multicolumn{4}{|l|}{ Question } \\
\hline 1. The trauma meeting follows a structured format each day & $7.7(2.1)$ & $6.7(2.2)$ & $7.5(1.5)$ \\
\hline $\begin{array}{l}\text { 2. The order of patients for the trauma list on that day is made clear } \\
\text { and apparent }\end{array}$ & $6.1(2.5)$ & $6.4(2.0)$ & $6.3(1.9)$ \\
\hline 4. The operation decided for each patient is clearly communicated & $6.7(2.6)$ & $7.6(2.1)$ & $7.4(1.8)$ \\
\hline 5. The operation decided for each patient is clearly documented & $5.5(3.1)$ & $6.5(1.7)$ & $7.5(1.4)$ \\
\hline 6. The plan for new referrals is clear & $7.1(2.0)$ & $7.4(1.5)$ & $7.3(1.3)$ \\
\hline 7. The plan for new referrals is documented & $6.0(2.6)$ & $6.5(2.0)$ & $7.1(1.8)$ \\
\hline
\end{tabular}

1-10 Likert scale. 1 = strongly disagree. 10 = strongly agree. 


\section{LESSONS AND LIMITATIONS}

This quality improvement project highlighted the benefits of introduction of a modern cloud-based trauma platform on both the quality of input referrals and the overall clarity and organisation of the trauma meeting. However, there were several lessons that were learnt and further improvements that could be made.

One of the main issues during our project was regarding resistance to change and familiarity with the new trauma system. Our initial reaudit at 1 month postintroduction of the new system showed no improvement in the clarity and structure of the trauma meeting. Authors found that there was often issues with the order of list printing creating confusion about which order patients were to be presented in the trauma meeting. Presentation at the audit meeting helped to establish a set order of list printing to resemble the previous order used with the old system. This helped provide a familiar structure to the trauma meeting. At 1 month, authors also noted that individuals were not utilising key aspects of the new system such as the ability to document management plans and change the trauma list order. These features were again highlighted to the team in the following months as well as at the audit presentation day, leading to improvement when reauditing at 4 months. This highlights the importance of familiarity and re-enforcement with incorporation of new electronic systems. We had further issues with accurate data coding for operations; this has since been highlighted to junior doctors and is regularly checked within the trauma meeting to ensure patients are listed (and coded) for the correct operation.

Any trauma meeting is only as good as the individual clinicians coordinating them; usually the on-call consultant and trauma nurses. It should be noted that no trauma system, no matter how good, can replace effective communication between members of the trauma team. Electronic trauma systems are a key organisational tool and visual aid with which to structure the trauma meeting around. In auditing qualitative opinions of the trauma meeting, we were inadvertently assessing these two modalities. First, the impact of the trauma system and second, the communication skills of the trauma team members. Therefore, the improvements seen may not be attributable to the new trauma system alone. There remains the possibility that in assessing the trauma meeting on a random selection of days the changes could simply amount to random variation of the team members present and their individual communication skills.

Our data collection combined both objective and subjective assessments, as seen with similar quality improvement projects, ${ }^{1819}$ producing more substantial evidence than objective or subjective assessments alone. The subjective element allowed us to assess quality improvement in terms of individual team members experience, while the objective measurements reduce the risk of bias and enables comparison of data values. We also analysed a large number of referrals at each reaudit cycle helping accommodate for any variation between the quality of different individual's data entry skills.

There were several limitations of this quality improvement. The first is our concern that an audit effect may account for some of the improvement following our presentation to members of the team, however, the effect this had on results is difficult to quantify. Second, we feel that all members of the team should have been distributed questionnaires at each stage, thus giving a more accurate representation of the whole trauma team and giving up larger numbers for comparison. Lastly, we should have included an evaluation of the teaching element of the trauma meeting alongside questions regarding the ease and speed of inputting referrals into each platform. These are the two areas we plan to look into with future prospective audits or quality improvement projects.

Our primary SMART aim was to improve the quality of our data entry in at least $50 \%$ of our predefined key data points, which we have successfully achieved. Inputting of patient demographics, ward location, bed allocation and responsible consultant are an essential function of an electronic trauma system. Both systems allowed inputting of these essential modalities, however, eTrauma users input the bed allocation of patients more frequently. Although improved with the new system, assignment of bed allocation remained at $49 \%$. On feedback from junior doctors, it was noted that often patients had not been assigned an inpatient bed number at time of inputting the referral, therefore at that time doctor's could not fill out this information on the system. Due to this and after discussion with the team we agreed that this should be updated prior to morning handover by the on-call Senior House Officer (SHO) and trauma nurses. Therefore, ensuring that the post-take ward round is well organised and runs in a coordinated fashion.

The eTrauma system showed better quality of data entry for 11 out of the 14 data points for referral details. Authors mainly attribute this finding to the improved layout of the eTrauma system with coded text boxes (figure 3) to input the following details: diagnosis, laterality, mechanism of injury, past medical history, allergies, drug history and social history including walking aids/baseline mobility. The data points that did not show improvement were diagnosis, laterality and blood results. The diagnosis and laterality were recorded $>94 \%$ of the time on TIGER and thus was unlikely to show significant improvement with the new system. It is interesting, however, that blood results were the only data point that we analysed that does not have a specific data entry text box on the new platform (see figure 3). Authors have since liaised with the eTrauma team to consider generation of a new input box for investigation and blood results.

Our secondary SMART aim was to improve the structure and quality of our trauma meeting by an improved response in $75 \%$ of our qualitative feedback questions. We showed improvements in $7(87.5 \%)$ out of the 8 questions at 4 months, therefore achieving our secondary aim. The most notable improvements were seen in the 
communication and documentation of operations to be performed as well as the clarity and documentation of management plans for each new referral. The questionnaires acted as a balancing measure for the study, to ensure that improvements in one aspect of the trauma meeting were not negatively impacting on another area.

With introduction and incorporation of the new trauma system into our departments working practice, authors feel that improvements seen within our project are sustainable. Reaudit at 4 months showed continued and sustained improvement which is an encouraging sign. One area of future research is to look at the cost analysis of the new trauma system, with the aim of comparing the initial cost of incorporating the new trauma system versus the potential gains in renumeration due to improved and more accurate data coding.

To conclude, introduction of a new cloud-based trauma management platform has had a positive impact overall within our trust. Significant improvements were seen with the quality of electronic trauma referrals, with increased data entry of several modalities that constitute a good orthopaedic referral. Alongside this, the trauma system had a positive impact on some aspects of the structure of the trauma meeting. There was increased clarity of the trauma list and better documentation of operations to be performed and management plans for new referrals. Modern cloud-based electronic trauma systems have the ability to improve our trauma management, however, this must go hand in hand with a structured and effectively communicated trauma meeting.

Twitter Benjamin Michael Sephton @benjaminsephton

Contributors All the authors have participated during the preparation and writing of the manuscript. All the authors have read and accepted the final format of the manuscript.

Funding The authors have not declared a specific grant for this research from any funding agency in the public, commercial or not-for-profit sectors.

Competing interests Senior authors MS and PM declare a competing interest in their employment by Open Medical Ltd.

Patient and public involvement Patients and/or the public were not involved in the design, or conduct, or reporting or dissemination plans of this research.

Patient consent for publication Not required.

Provenance and peer review Not commissioned; externally peer reviewed.

Data availability statement Data are available upon request.

Open access This is an open access article distributed in accordance with the Creative Commons Attribution Non Commercial (CC BY-NC 4.0) license, which permits others to distribute, remix, adapt, build upon this work non-commercially, and license their derivative works on different terms, provided the original work is properly cited, appropriate credit is given, any changes made indicated, and the use is non-commercial. See: http://creativecommons.org/licenses/by-nc/4.0/.

\section{ORCID iD}

Benjamin Michael Sephton http://orcid.org/0000-0002-8058-168X

\section{REFERENCES}

1 Health \& Social Care Information Centre. Accident and emergency attendances in England (experimental statistics) 2007-2008. NHS England: HSCIC, 2009.

2 National Institute for Health and Care Excellence. National Institute for health and clinical excellence. fractures (non-complex): assessment and management. Available: https://www.nice.org.uk [Accessed 4 Jun 2020].

3 General Medical Council. National training survey 2014 key findings, 2014. Available: http://www.gmc-uk.org/NTS_2014_KFR_A4.pdf_ 56706809.pdf

4 Freemantle N, Richardson M, Wood J, et al. Weekend hospitalization and additional risk of death: an analysis of inpatient data. J $R$ Soc Med 2012;105:74-84.

5 The Royal College of Surgeons of England. The higher risk general surgical patient: towards improved care for a forgotten group. London: RCSE, 2011. http://www.rcseng.ac.uk/publications/docs/ higher-risk-surgical-patient

6 Institution for Innovation and Improvement. Improving quality and efficiency in the operating theatre [Internet]. Coventry: NHS. Available: http://harmfreecare.org/wp-content/files_mf/Improvingquality-and-efficiency-in-the-operating-theatre.pdf

7 Mohan A, Lutterodt C, Leon-Villapalos J. Operating efficiency of an emergency burns theatre: an eight month analysis. Burns 2017;43:1435-40.

8 Ang WW, Sabharwal S, Johannsson $\mathrm{H}$, et al. The cost of trauma operating theatre inefficiency. Ann Med Surg 2016;7:24-9.

9 Govier M, Medcalf P. Living for the weekend: electronic documentation improves patient handover. Clin Med 2012;12:124-7.

10 Till A, Sall H, Wilkinson J. Safe Handover : Safe Patients - The Electronic Handover System. BMJ Qual Improv Rep 2014;2. doi:10.1136/bmjquality.u202926.w1359. [Epub ahead of print: 26 Feb 2014].

11 Karayiannis P, Warnock J. Improving handover of acute orthopaedic admissions. BMJ Qual Improv Rep 2015;4:u209209.w3901.

12 Dean J, Phillips G, Turner W, et al. Demonstrating improved surgical communication and handover generates earlier discharges (discharged). J Patient Saf 2017;1.

13 Bhabra G, Mackeith S, Monteiro P, et al. An experimental comparison of handover methods. Ann R Coll Surg Engl 2007;89:298-300.

14 British Medical Association. Safe handover: safe patients. guidance on clinical handover for clinicians and managers. London: BMA, 2004.

15 Royal College of Surgeons of England. Safe handover: guidance from the working time directive Working Party. London: Royal College of Surgeons of England, 2007.

16 Royal College of Physicians. Acute care toolkit 1: handover. Royal College of Physicians, 2011.

17 Allen IE, Seaman CA. Likert scales and data analyses. Qual Prog 2007;40:64-5.

18 Saifuddin A, Magee L, Barrett R, et al. "Chase CRP", "Review patient": Improving the Quality of Weekend Medical Handover at a London Teaching Hospital. BMJ Qual Improv Rep 2015;4. doi:10.1136/bmjquality.u201656.w1919. [Epub ahead of print: 24 Nov 2015].

19 Jardine AGM, Page T, Bethune R, et al. Bring on the weekend Improving the quality of junior doctor weekend handover. BMJ Qual Improv Rep 2014;2:u202379.w1297. 